\section{Nierenkranke Kinder}

\section{Strikte Blutdruckeinstellung reduziert Dialyserisiko um 35\%}

— Die Ergebnisse einer europäischen Studie zeigen jetzt erstmals, dass bei chronisch nierenkranken Kindern die Nierenfunktion länger erhalten bleibt, wenn der Blutdruck der Kinder konsequent abgesenkt wird.

„Aus Studien an Erwachsenen weiß man, dass ACE-Hemmer die Nieren schützen können. Bislang fehlten jedoch zuverlässige Daten für eine Therapie beim Kind“, erklärt Prof. Schaefer, Leiter der Studie vom Universitätsklinikum Heidelberg [N Engl J Med 2009; 361 (17): 1639-50] in einer Pressemitteilung der Klinik.

An der ESCAPE-Studie (Effect of Strict blood pressure Control and ACE inhibition on the progression of chronic renal failure in PEdiatric patients) nahmen 385 nierenkranke Kinder in 33 europäischen Behandlungszentren teil. Während in der konventionell behandelten Gruppe, bei der die Blutdruckwerte im oberen Normalbereich eingestellt wurden, nach 5 Jahren $41,7 \%$ der Kinder eine Nierenersatztherapie benötigten, waren dies in der intensiviert behandelten Gruppe mit Zielwert im unteren Normalbereich nur 29,9\% der Patienten. Beide Gruppen erhielten einen ACE-Hemmer und, falls notwendig, weitere blutdrucksenkende Medikamente anderer Stoffklassen. Obwohl sich die therapeutisch erzielten Blutdruckwerte der beiden Gruppen nur leicht unterschieden $(3-4 \mathrm{mmHg}$ ), waren die Ergebnisse signifikant besser mit der intensivierten Therapie.

„Unsere Ergebnisse zeigen, dass eine konsequente, strenge Blutdruckeinstellung das Risiko des endgültigen Nierenversagens um 35\% senken kann“. erklärte PD Dr. Elke Wühl von der Heidelberger Arbeitsgruppe. „Besonders gut stehen die Chancen eine Nierenersatztherapie aufschieben zu können, wenn nicht nur die angestrebten Blutdruckwerte erreicht werden, sondern zu Behandlungsbeginn auch eine Abnahme der häufig erhöhten Eiweißausscheidung im Urin erzielt werden kann. “

uls

\section{Prostatarezidive erkennen und behandeln}

— Die Entstehung von Prostatatumoren könnte auf der Fehlfunktion bestimmter Stammzellen beruhen, berichten Wissenschaftler vom Columbia University Medical Center in New York [Wand X et al. Nature 2009; 461: 495-500]. Die sogenannten Castration-Resistant-Nkx3-1-Expressing Cells (CARNs) finden sich im Epithel, das die Innenseite der Drüse auskleidet.

Urologen der Mayo-Klinik in Jacksonville, Florida/USA plädieren für die „Salvage-Radiotherapie“ bei PSA-rezidivierten prostatektomierten Männern. Die Wissenschaftler um Jennifer Peterson führten die nach Klinikangaben größte unizentrische Studie zu diesem Thema durch, an der 308 Patienten mit einem medianen Follow-up von 6o Monaten nach der Radiotherapie teilnahmen. Nebenwirkungen vom Grad 3 oder 4 traten nur bei 3 bzw. 1 Patienten auf. Wie die Klinik mitteilt, erscheint die Studie demnächst im Fachmagazin Radiotherapy \& Oncology.

Um PSA-Rezidiven zuverlässiger auf die Spur zu kommen, haben Biochemiker der Northwestern University in Evanston/Illinois/USA ein Nanotestsystem entwickelt. Es soll nahezu 300-fach sensitiver sein als derzeit verfügbare PSATests [Thaxton CS et al. PNAS, online vorab 19.10.2009].

\section{Prostatakarzinom}

\section{Knochenschwund muss nicht sein}

— Patienten mit nicht-metastasiertem Prostatakarzinom profitieren eindeutig von einer Androgendeprivationstherapie. Der Preis dafür ist allerdings ein Verlust an Knochenmasse. Dem lässt sich entgegensteuern, wie eine aktuelle Studie zeigt.

In der randomisierten Doppelblindstudie waren je 734 Patienten entweder alle sechs Monate mit einer subkutanen $60 \mathrm{mg}$-Dosis des monoklonalen Antikörpers Denosumab oder Placebo behandelt worden [Smith R et al. N Engl J Med 2009; 361: 745-55]. Der monoklonale Antikörper ist gegen RANKL (Receptor Activator of nuclear factor-B ligand), einen wichtigen Botenstoff der Kommunikation zwischen Osteoblasten und Osteoklasten gerichtet und verhindert so die Bindung von RANKL an seinen Rezeptor auf Präosteoklasten. Dies führt letztendlich zu einer verminderten Knochenresorption.

Bereits nach einem Monat gab es signifikante Knochendichte-Unterschiede zwischen beiden Gruppen; sie hielten für den gesamten Studienzeitraum von 36 Monaten an. Nach 24 Monaten hatte die Knochendichte im Bereich der Lendenwirbelsäule unter Denosumab um $5,6 \%$ zugenommen, unter Placebo um $1 \%$ abgenommen. Auch an Hüfte, Femurkopf und distalem Drittel des Radius war die Knochendichte in der Denosumab-Gruppe signifikant höher. Nach 36 Monaten schlug sich die Behandlung mit dem monoklonalen Antikörper in einer Senkung der Rate vertebraler Frakturen um 62\% nieder (1,5 versus 3,9\%). Die Rate unerwünschter Nebenwirkungen war nicht erhöht.

wk

\title{
Portal zur Kassenbewertung
}

- Als Pendant zum Arztbewertungsportal „AOKArzt-Navigator", das die AOK 2010 starten will, hat der Krefelder Allgemeinarzt Dr. Norbert A. Scholz einen Webauftritt zur Bewertung von Kassen ins Leben gerufen. Auf dem Onlineportal [www.krankenkassenbewertung.net] können Ärzte und andere Vertreter aus dem Gesundheitsbereich, aber auch Versicherte selbst Krankenkassen benoten und ihre Erfahrungen austauschen. Hierzu muss ein kurzer Fragebogen ausgefüllt werden, die Daten des Bewerters werden dabei jedoch nicht veröffentlicht.

nbr 